\title{
Mathematical Overview of Hypersphere World-Universe Model
}

\author{
Vladimir S. Netchitailo \\ Biolase Inc., Irvine CA, USA \\ Email:v.netchitailo@sbcglobal.net
}

How to cite this paper: Netchitailo, V.S. (2017) Mathematical Overview of Hypersphere World-Universe Model. Journal of High Energy Physics, Gravitation and Cosmology, 3, 415-437.

https://doi.org/10.4236/jhepgc.2017.33033

Received: March 30, 2016

Accepted: June 6, 2017

Published: June 9, 2017

Copyright $\odot 2017$ by author and Scientific Research Publishing Inc. This work is licensed under the Creative Commons Attribution International License (CC BY 4.0).

http://creativecommons.org/licenses/by/4.0/ (c) (i) Open Access

\begin{abstract}
The Hypersphere World-Universe Model (WUM) provides a mathematical framework that allows calculating the primary cosmological parameters of the World which are in good agreement with the most recent measurements and observations. WUM explains the experimental data accumulated in the field of Cosmology and Astroparticle Physics over the last decades: the age of the World and critical energy density; the gravitational parameter and Hubble's parameter; temperatures of the cosmic microwave background radiation and the peak of the far-infrared background radiation; the concentration of intergalactic plasma and time delay of Fast Radio Bursts. Additionally, the model predicts masses of dark matter particles, photons, and neutrinos; proposes new types of particle interactions (Super Weak and Extremely Weak); shows inter-connectivity of primary cosmological parameters of the World. WUM proposes to introduce a new fundamental parameter $Q$ in the CODATA internationally recommended values. This paper is the summary of the mathematical results obtained in [1]-[4].
\end{abstract}

\section{Keywords}

Hypersphere World-Universe Model, Primary Cosmological Parameters,

Medium of the World, Macroobjects Structure, Gravitoelectromagnetism, Dark Matter Particles, Intergalactic Plasma, Microwave Background

Radiation, Far-Infrared Background Radiation, Fast Radio Bursts,

Emergent Phenomena, CODATA

\section{Introduction}

Hypersphere World-Universe Model (WUM) views the World as a 3-dimensional hypersphere that expands along the fourth spatial dimension in the Universe. A hypersphere is an example of a 3-manifold which locally behaves like regular euclidean 3-dimensional space: just as a sphere looks like a plane to small 
enough observers. WUM is based on Maxwell's equations (ME) that form the foundation of Electromagnetism and Gravitoelectromagnetism. According to ME, there exist two measurable physical characteristics: energy density and energy flux density.

WUM makes reasonable assumptions in the main areas of cosmology. The remarkable agreement of the calculated values of the primary cosmological parameters with the observational data gives us considerable confidence in the model.

The principal idea of WUM is that the energy density of the World $\rho_{W}$ equals to the critical energy density $\rho_{c r}$ necessary for 3-manifold at any cosmological time. $\rho_{c r}$ can be found by considering a sphere of radius $R_{M}$ and enclosed mass $M$, with a small test mass $m$ on the periphery of the sphere. Mass $M$ can be calculated by multiplication of $\rho_{c r}$ by the volume of the sphere. The equation for $\rho_{c r}$ can be found from the escape speed calculation for test mass $m:$

$$
\rho_{c r}=\frac{3 H^{2} c^{2}}{8 \pi G}
$$

where $G$ is the gravitational constant, $H$ is Hubble's parameter, and $c$ is the gravitoelectrodynamic constant that is identical to the electrodynamic constant $c$ in Maxwell's equations.

WUM introduces a fundamental dimensionless time-varying parameter $Q$ that is the measure of the curvature of the Hypersphere. $Q$ can be calculated from the average value of the gravitational constant and in present epoch equals to (see Section 2):

$$
Q=0.759972 \times 10^{40}
$$

WUM develops a mathematical framework that allows for direct calculation of a number of cosmological parameters through $Q$. The precision of such parameters increases by orders of magnitude (see Section 2). Below we will use the following fundamental constants:

- Basic unit of length $a=2 \pi a_{0}, a_{0}$ being the classical electron radius;

- Planck constant $h$;

- Basic unit of energy $E_{0}=\frac{h c}{a}$ that is the basic gravitoelectrodynamic charge;

- Basic unit of energy density $\rho_{0}=\frac{h c}{a^{4}}$;

- Basic unit or surface energy density $\sigma_{0}=\frac{h c}{a^{3}}=\rho_{0} a$;

- Basic unit of mass $m_{0}=\frac{h}{a c}$;

- Basic unit of frequency $v_{0}=\frac{c}{a}$;

- Fine-structure constant $\alpha$.

\section{Primary Cosmological Parameters}

Equation (1.1) can be rewritten as 


$$
\frac{4 \pi G}{c^{2}} \times \frac{2}{3} \rho_{c r}=\mu_{g} \times \rho_{M}=H^{2}
$$

where $\mu_{g}$ is the gravitomagnetic parameter and $\rho_{M}$ is the energy density of the Medium. Hubble's parameter $H$ can be expressed: $H=\frac{C}{R}$, where $R$ is the Hubble's radius and is the radius of the Hypersphere in WUM. Introducing the dimensionless parameter $Q$.

$$
Q=\frac{R}{a}=v_{0} H^{-1}
$$

we can rewrite (2.1)

$$
\frac{8 \pi G a^{2}}{c^{4}} \times \frac{1}{3} \rho_{c r}=\frac{8 \pi G a^{2}}{c^{4}} \times \rho_{M O}=\frac{8 \pi G a^{2} \rho_{0}}{c^{4}} \times \frac{\rho_{M O}}{\rho_{0}}=Q^{-2}
$$

where $\rho_{M O}$ is the energy density of Macroobjects of the World. Assuming that

$$
\rho_{\text {MO }}=\rho_{0} \times Q^{-1}
$$

we can find the equation for the critical energy density:

$$
\rho_{c r}=3 \rho_{0} \times Q^{-1}
$$

and for the gravitational constant:

$$
G=\frac{a^{3} c^{3}}{8 \pi h c} H=\frac{a^{2} c^{4}}{8 \pi h c} \times Q^{-1}
$$

We can calculate the value of $G$ based on the value of $H$. Conversely, we can find the value of the Hubble's parameter based on the value of the gravitational parameter. $H$ and $G$ are interchangeable! Knowing value of one, it is possible to calculate the other.

According to (2.2) we can find the value of dimensionless parameter $Q$ based on the value of $H$, but the accuracy of its measurements is very poor. We have obtained the value of $Q$ in (1.2) based on the Equation (2.6), and value of $G$ that is measured with much better accuracy. Then we can calculate the value of $H_{0}$ in present epoch:

$$
H_{0}=v_{0} Q^{-1}=68.7457(83) \mathrm{km} / \mathrm{s} \cdot \mathrm{Mpc}^{-1}
$$

Thus calculated value of $H_{0}$ is in excellent agreement with experimentally measured value of $H_{0}=69.32 \pm 0.8 \mathrm{~km} / \mathrm{s} \cdot \mathrm{Mpc}^{-1}$ [5] and proves assumption (2.4).

\section{Gravitation}

In frames of WUM the parameter $G$ can be calculated based on the value of the energy density of the Medium $\rho_{M}$ [2]:

$$
G=\frac{\rho_{M}}{4 \pi} \times P^{2}
$$

where a dimension-transposing parameter $P$ equals to:

$$
P=\frac{a^{3}}{2 h / c}
$$


Then the Newton's law of universal gravitation can be rewritten in the following way:

$$
F=G \frac{m \times M}{r^{2}}=\frac{\rho_{M}}{4 \pi} \frac{\frac{a^{3}}{2 L_{C m}} \times \frac{a^{3}}{2 L_{C M}}}{r^{2}}
$$

where we introduced the measurable parameter of the Medium $\rho_{M}$ instead of the phenomenological coefficient $G$; and gravitoelectromagnetic charges $\frac{a^{3}}{2 L_{C m}}$ and $\frac{a^{3}}{2 L_{C M}}$ instead of macroobjects masses $m$ and $M\left(L_{C m}\right.$ and $L_{C M}$ are Compton length of mass $m$ and $M$ respectively). The gravitoelectromagnetic charges in (3.3) have a dimension of "Area", which is equivalent to "Energy", with the constant that equals to the basic unit of surface energy density $\sigma_{0}$.

Following the approach developed in [2] we can find the gravitomagnetic parameter of the Medium $\mu_{M}$ :

$$
\mu_{M}=R^{-1}
$$

and the impedance of the Medium $Z_{M}$ :

$$
Z_{M}=\mu_{M} c=H=\tau^{-1}
$$

where $\tau$ is a cosmological time. These parameters are analogous to the permeability $\mu_{0}$ and impedance of electromagnetic field $Z_{0}=\sqrt{\frac{\mu_{0}}{\varepsilon_{0}}}=\mu_{0} c$, where $\varepsilon_{0}$ is the permittivity of electromagnetic field and $\mu_{0} \varepsilon_{0}=c^{-2}$.

It follows that measuring the value of Hubble's parameter anywhere in the World and taking its inverse value allows us to calculate the absolute Age of the World. The Hubble's parameter is then the most important characteristic of the World, as it defines the Worlds' Age. While in our Model Hubble's parameter $H$ has a clear physical meaning, the gravitational parameter $G=\frac{c^{3}}{8 \pi \sigma_{0}} H$ is a phenomenological coefficient in the Newton's law of universal gravitation.

The second important characteristic of the World is the gravitomagnetic parameter $\mu_{M}$. Taking its inverse value, we can find the absolute radius of curvature of the World in the fourth spatial dimension. We emphasize that the above two parameters $\left(Z_{M}\right.$ and $\left.\mu_{M}\right)$ are principally different physical characteristics of the Medium that are connected through the gravitoelectrodynamic constant $c$. It means that Time is not a physical dimension and is absolutely different entity than Space. Time is a factor of the World.

It follows that Gravity, Space and Time itself can be introduced only for a World filled with Matter consisting of elementary particles which take part in simple interactions at a microscopic level. The collective result of their interactions can be observed at a macroscopic level. Gravity, Space and Time are then emergent phenomena [3]. 


\section{Intergalactic Plasma}

In our Model, the World consists of stable massive elementary particles with lifetimes longer than the age of the World. Protons with mass $m_{p}$ and energy $E_{p}=m_{p} c^{2}$ and electrons with mass $m_{e}$ and energy $E_{e}=m_{e} c^{2}=\alpha E_{0}$ have identical concentrations in the World: $n_{p}=n_{e}$.

Low density intergalactic plasma consisting of protons and electrons has plasma frequency $\omega_{p l}$ :

$$
\omega_{p l}^{2}=\frac{4 \pi n_{e} e^{2}}{4 \pi \varepsilon_{0} m_{e}}=4 \pi n_{e} \alpha \frac{h}{2 \pi m_{e} c} c^{2}=2 n_{e} a c^{2}
$$

where $e$ is the elementary charge. Since the formula calculating the potential energy of interaction of protons and electrons contains the same parameter $k_{p e}$ :

$$
k_{p e}=m_{p} \omega_{p l}^{2}=m_{e} \omega_{e}^{2}=m_{e}\left(2 \pi v_{0} \times Q^{-1 / 2}\right)^{2}
$$

where we assume that $\omega_{e}$ is proportional to $Q^{-1 / 2}$, then $\omega_{p l}^{2}$ is proportional to $Q^{-1}$. Energy densities of protons and electrons are then proportional to $Q^{-1}$, similar to the critical energy density $\rho_{c r} \propto Q^{-1}$.

We substitute $\omega_{p l}^{2}=\frac{m_{e}}{m_{p}}\left(2 \pi v_{0} \times Q^{-1 / 2}\right)^{2}$ into (4.1) and calculate concentration of protons and electrons:

$$
n_{p}=n_{e}=\frac{2 \pi^{2}}{a^{3}} \frac{m_{e}}{m_{p}} \times Q^{-1}=0.25480 \mathrm{~m}^{-3}
$$

A. Mirizzi, et al. found that the mean diffuse intergalactic plasma density is bounded by $n_{e} \lesssim 0.27 \mathrm{~m}^{-3}$ [6] corresponding to the WMAP measurement of the baryon density [7]. The Mediums' plasma density (4.3) is in good agreement with the estimated value [6].

From Equation (4.2) we obtain the value of the lowest frequency $v_{p l}$ :

$$
v_{p l}=\frac{\omega_{p l}}{2 \pi}=\left(\frac{m_{e}}{m_{p}}\right)^{1 / 2} v_{0} \times Q^{-1 / 2}=4.5322 \mathrm{~Hz}
$$

Photons with energy smaller than $E_{p h}=h v_{p l}$ cannot propagate in plasma, thus $h v_{p l}$ is the smallest amount of energy a photon may possess. Following the authors of [8] we can call this amount of energy the rest energy of photons that equals to

$$
E_{p h}=\left(\frac{m_{e}}{m_{p}}\right)^{1 / 2} \times E_{0} \times Q^{-1 / 2}=1.8743 \times 10^{-14} \mathrm{eV}
$$

The above value is in good agreement with the value $E_{p h} \lesssim 2.2 \times 10^{-14} \mathrm{eV}$ estimated in [8]. It is more relevant to call $E_{p h}$ the minimum energy of photons which can pass through the Intergalactic plasma.

$\rho_{p}=n_{p} E_{p}$ is the energy density of protons in the Medium. The relative energy density of protons $\Omega_{p}$ is then the ratio of $\rho_{p} / \rho_{c r}$ :

$$
\Omega_{p}=\frac{\rho_{p}}{\rho_{\text {cr }}}=\frac{2 \pi^{2} \alpha}{3}=0.048014655
$$


This value is in good agreement with experimentally found value of $0.049 \pm 0.013$ [9]. The results obtained in [6] [8] and [9] prove assumption (4.2).

According to WUM, the black body spectrum of Microwave Background Radiation (MBR) is due to thermodynamic equilibrium of photons with low density intergalactic plasma consisting of protons and electrons. $\rho_{e}=n_{e} E_{e}$ is the energy density of electrons in the Medium. We assume that the energy density of MBR $\rho_{M B R}$ equals to twice the value of $\rho_{e}$ :

$$
\rho_{M B R}=2 \rho_{e}=4 \pi^{2} \alpha \frac{m_{e}}{m_{p}} \rho_{0} \times Q^{-1}=\frac{8 \pi^{5}}{15} \frac{k_{B}^{4}}{(h c)^{3}} T_{M B R}^{4}
$$

where $k_{B}$ is the Boltzmann constant and $T_{M B R}$ is MBR temperature. We can now calculate the value of $T_{M B R}$ :

$$
T_{M B R}=\frac{E_{0}}{k_{B}}\left(\frac{15 \alpha}{2 \pi^{3}} \frac{m_{e}}{m_{p}}\right)^{1 / 4} \times Q^{-1 / 4}=2.72518 \mathrm{~K}
$$

Thus calculated value of $T_{M B R}$ is in excellent agreement with experimentally measured value of $2.72548 \pm 0.00057 \mathrm{~K} \quad$ [10] and proves assumption (4.7).

\section{Fast Radio Bursts}

Fast Radio Burst (FRB) is a high-energy astrophysical phenomenon manifested as a transient radio pulse lasting only a few milliseconds. These are bright, unresolved, broadband, millisecond flashes found in parts of the sky outside the Milky Way. The component frequencies of each burst are delayed by different amounts of time depending on the wavelength. This delay is described by a value referred to as a Dispersion Measure (DM) which is the total column density of free electrons between the observer and the source of FRB. Fast radio bursts have DMs which are: much larger than expected for a source inside the Milky Way [11]; and consistent with propagation through ionized plasma [12]. In this Section we calculate a time delay of FRB based on the characteristics of the Intergalactic Plasma discussed in [4] (see Section 4).

Consider a photon with initial frequency $v_{\text {emit }}$ and energy $E_{\text {emit }}$ emitted at time $\tau_{\text {emit }}$ when the radius of the hypersphere World in the fourth spatial dimension was $R_{\text {emit }}$. The photon is continuously losing kinetic energy as it moves from galaxy to the Earth until time $\tau_{\text {obsv }}$ when the radius is $R_{\text {obsv }}=R_{0}$. The observer will measure $v_{\text {obsv }}$ and energy $E_{\text {obsv }}$ and calculate a redshift:

$$
1+z=\frac{v_{\text {emit }}}{v_{\text {obsv }}}=\frac{E_{\text {emit }}}{E_{\text {obsv }}}
$$

Recall that $\tau_{\text {emit }}$ and $\tau_{\text {obsv }}$ are cosmological times (ages of the World at the moments of emitting and observing). A light-travel time distance to a galaxy $d_{L T T}$ equals to

$$
d_{L T T}=c\left(\tau_{\text {obsv }}-\tau_{\text {emit }}\right)=c t_{L T T}=R_{0}-R_{\text {emit }}
$$

Let's calculate photons' traveling time $t_{p h}$ from a galaxy to the Earth taking 
into account that the rest energy of photons $E_{p h}$ is much smaller than the energy of photons $E_{\gamma}: E_{p h} \ll E_{\gamma}$.

$$
t_{p h}=\frac{1}{c} \int_{R_{e m i t}}^{R_{0}} \frac{\mathrm{d} r}{\sqrt{1-\frac{E_{p h}^{2}}{E_{\gamma}^{2}}}}=t_{L T T}+\Delta t_{p h}
$$

where $\Delta t_{p h}$ is photons' time delay relative to the light-travel time $t_{L T T}$ that equals to:

$$
\Delta t_{p h}=\frac{1}{2 c} \int_{R_{\text {emit }}}^{R_{0}} \frac{E_{p h}^{2}}{E_{\gamma}^{2}} \mathrm{~d} r
$$

All observed FRBs have redshifts $z<1$. It means that we can use the Hubble's law: $d_{L T T}=R_{0} z$. Then

$$
R_{\text {emit }}=(1-z) R_{0}
$$

Photons' rest energy squared at radius $r$ between $R_{\text {emit }}$ and $R_{0}$ equals to (3.5):

$$
E_{p h}^{2}=\frac{m_{e}}{m_{p}} \frac{a}{r} E_{0}^{2}
$$

According to WUM, photons' energy $E_{\gamma}$ on the way from galaxy to an observer can be expressed by the following equation:

$$
E_{\gamma}=z E_{\text {obsv }}+(1-z) \frac{R_{0}}{r} E_{\text {obsv }}=z \frac{R_{0}}{r} E_{\text {obsv }}\left(\frac{1-z}{z}+\frac{r}{R_{0}}\right)
$$

which reduces to $E_{\text {emit }}$ at (5.5) and to $E_{\text {obsv }}$ at $r=R_{0}$. Placing the values of the parameters (5.5), (5.6), (5.7) into (5.4), we have for photons' time delay:

$$
\begin{aligned}
\Delta t_{p h} & =\frac{1}{2 z^{2}} \frac{c}{a} \frac{m_{e}}{m_{p}} \frac{1}{v^{2}} \int_{1-z}^{1} \frac{x \mathrm{~d} x}{\left(x+\frac{1-z}{z}\right)^{2}} \\
& =\frac{1}{2 z^{2}} \frac{c}{a} \frac{m_{e}}{m_{p}} \frac{1}{v^{2}} \int_{\frac{1-z^{2}}{z}}^{\frac{1}{z}} \frac{\left(y-\frac{1-z}{z}\right) d y}{y^{2}} \\
& =\frac{1}{2 z^{2}}\left[\ln \left(\frac{1}{1-z^{2}}\right)-\frac{z^{2}}{1+z}\right] \frac{c}{a} \frac{m_{e}}{m_{p}} \times \frac{1}{v^{2}} \\
& =\frac{4.61}{z^{2}}\left[\ln \left(\frac{1}{1-z^{2}}\right)-\frac{z^{2}}{1+z}\right] \times\left(\frac{v}{1 \mathrm{GHz}}\right)^{-2}
\end{aligned}
$$

where $x=r / R_{0}$ and $y=x+\frac{1-z}{z}$. Taking $z=0.492$ [12] we get the calculated value of photons' time delay

$$
\Delta t_{p h}^{c a l}=2.189 \times\left(\frac{v}{1 \mathrm{GHz}}\right)^{-2}
$$

which is in good agreement with experimentally measured value [12]

$$
\Delta t_{p h}^{e x p}=2.438 \times\left(\frac{v}{1 \mathrm{GHz}}\right)^{-2}
$$


It is worth to note that in our calculations there is no need in the dispersion measure.

\section{Neutrinos}

It is now established that there are three different types of neutrino: electronic $v_{e}$, muonic $v_{\mu}$, and tauonic $v_{\tau}$, and their antiparticles. Neutrino oscillations imply that neutrinos have non-zero masses [13] [14].

Let's take neutrino masses $m_{v_{e}}, m_{v_{\mu}}, m_{v_{\tau}}$ that are near [15]

$$
m_{v}=m_{0} \times Q^{-1 / 4}
$$

Their concentrations $n_{V}$ are then proportional to

$$
n_{v} \propto \frac{1}{a^{3}} \times Q^{-3 / 4}
$$

and energy densities of neutrinos are proportional to $Q^{-1}$, since critical energy density $\rho_{c r}$ is proportional to $Q^{-1}$ (see Section 2).

Experimental results obtained by $\mathrm{M}$. Sanchez [16] show $v_{e} \rightarrow v_{\mu, \tau}$ neutrino oscillations with parameter $\Delta m_{\text {sol }}^{2}$ given by

$$
2.3 \times 10^{-5} \mathrm{eV}^{2} / \mathrm{c}^{4} \leq \Delta m_{\text {sol }}^{2} \leq 9.3 \times 10^{-5} \mathrm{eV}^{2} / \mathrm{c}^{4}
$$

and $v_{\mu} \rightarrow v_{\tau}$ neutrino oscillations with parameter $\Delta m_{a t m}^{2}$ :

$$
1.6 \times 10^{-3} \mathrm{eV}^{2} / \mathrm{c}^{4} \leq \Delta m_{a t m}^{2} \leq 3.9 \times 10^{-3} \mathrm{eV}^{2} / \mathrm{c}^{4}
$$

where $\Delta m_{\text {sol }}^{2}$ and $\Delta m_{\text {atm }}^{2}$ are mass splitting for solar and atmospheric neutrinos respectively. Significantly more accurate result was obtained by P. Kaus, et al. [17] for the ratio of the mass splitting:

$$
\sqrt{\frac{\Delta m_{\text {sol }}^{2}}{\Delta m_{\text {atm }}^{2}}} \cong 0.16 \approx \frac{1}{6}
$$

Let's assume that muonic neutrino's mass indeed equals to

$$
m_{v_{\mu}}=m_{v}=m_{0} \times Q^{-1 / 4} \cong 7.5 \times 10^{-3} \mathrm{eV} / \mathrm{c}^{2}
$$

From equation (6.5) it then follows that

$$
m_{v_{\tau}}=6 m_{v} \cong 4.5 \times 10^{-2} \mathrm{eV} / \mathrm{c}^{2}
$$

Then the squared values of the muonic and tauonic neutrino masses fall into ranges (6.3) and (6.4):

$$
\begin{aligned}
& m_{v_{\mu}}^{2} \cong 5.6 \times 10^{-5} \mathrm{eV}^{2} / \mathrm{c}^{4} \\
& m_{v_{\tau}}^{2} \cong 2 \times 10^{-3} \mathrm{eV}^{2} / \mathrm{c}^{4}
\end{aligned}
$$

Let's assume that electronic neutrino mass equals to

$$
m_{v_{e}}=\frac{1}{24} m_{v} \cong 3.1 \times 10^{-4} \mathrm{eV} / \mathrm{c}^{2}
$$

The sum of the calculated neutrino masses

$$
\sum m_{v} \cong 0.053 \mathrm{eV} / \mathrm{c}^{2}
$$

is also in a good agreement with the value of $0.06 \mathrm{eV} / \mathrm{c}^{2}$ discussed in literature 
[18].

Considering that all elementary particles, including neutrinos, are fully characterized by their four-momentum $\left(\frac{E_{v i}}{c}, \boldsymbol{p}_{v i}\right)$ :

$$
\left(\frac{E_{v i}}{c}\right)^{2}-\boldsymbol{p}_{v i}^{2}=\left(m_{v i} c\right)^{2}, i=e, \mu, \tau
$$

we obtain the following neutrino energy densities $\rho_{v i}$ in accordance with theoretical calculations made by L. D. Landau and E. M. Lifshitz [19]:

$$
\rho_{v i}=\frac{8 \pi c}{h^{3}} \int_{0}^{p_{F}} p^{2} \sqrt{p^{2}+m_{v i}^{2} c^{2}} \mathrm{~d} p=\frac{2 \pi\left(p_{F} c\right)^{4}}{(h c)^{3}} \times F\left(x_{v i}\right)
$$

where $p_{F}$ is Fermi momentum

$$
\begin{gathered}
F\left(x_{v i}\right)=\frac{x_{v i}^{1 / 2}\left(2 x_{v i}+1\right)\left(x_{v i}+1 / 2\right)^{1 / 2}-\ln \left[x_{v i}^{1 / 2}+\left(x_{v i}+1\right)^{1 / 2}\right]}{2 x_{v i}^{2}} \\
x_{v i}=\left(\frac{p_{F}}{m_{v i} c}\right)^{2} \\
m_{v i}=A_{i} m_{0} \times Q^{-1 / 4} \\
A_{i}=\frac{1}{24} ; 1 ; 6
\end{gathered}
$$

Let's take the following value for Fermi momentum $p_{F}$ :

$$
p_{F}^{2}=\frac{h^{2}}{2 \pi^{2} a^{2}} \times Q^{-1 / 2}=p_{F 0}^{2} \times Q^{-1 / 2}
$$

where $p_{F 0}^{2}=\frac{h^{2}}{2 \pi^{2} a^{2}}$ is the extrapolated value of $p_{F}$ at the Beginning when $Q=1$. Using (6.13), we obtain neutrinos relative energy densities $\Omega_{v i}$ in the Medium in terms of the critical energy density $\rho_{c r}$ :

$$
\Omega_{v i}=\frac{\rho_{v i}}{\rho_{c r}}=\frac{1}{6 \pi^{3}} F\left(y_{v i}\right)
$$

where

$$
y_{v i}=\left(2 \pi^{2} A_{i}^{2}\right)^{-1}
$$

It's commonly accepted that concentrations of all types of neutrinos are equal. This assumption allows us to calculate the total neutrinos relative energy density in the Medium:

$$
\Omega_{v}=\frac{\rho_{v}}{\rho_{c r}}=\frac{\rho_{v e}+\rho_{v \mu}+\rho_{v \tau}}{\rho_{c r}}=0.45801647
$$

One of the principal ideas of WUM holds that energy densities of Medium particles are proportional to proton energy density in the World's Medium [2]:

$$
\Omega_{p}=\frac{2 \pi^{2} \alpha}{3}=0.048014655
$$

which depends on the Fine-structure constant $\alpha$. We take the value of $\Omega_{v}$ to 
equal

$$
\Omega_{v}=\frac{30}{\pi} \Omega_{p}=20 \pi \alpha=0.45850618
$$

which is remarkably close to its value calculated in (6.20).

The assumptions made in (6.6), (6.9), (6.17) and (6.22) are further supported by the excellent numerical agreement of calculated and measured value of Fine-structure constant $\alpha$ discussed in Section 11.

\section{Cosmic Far-Infrared Background}

The cosmic Far-Infrared Background (FIRB), which was announced in January 1998, is part of the Cosmic Infrared Background, with wavelengths near $100 \mathrm{mi}$ crons that is the peak power wavelength of the black body radiation at temperature $29 \mathrm{~K}$. In this Section we introduce Bose-Einstein Condensate (BEC) drops of dineutrinos whose mass is about Planck mass, and their temperature is around $29 \mathrm{~K}$. These drops are responsible for the FIRB [15].

According to [20] [21] [22], the size of large cosmic grains $D_{G}$ is roughly equal to the length $L_{F}$ :

$$
D_{G} \sim L_{F}=a \times Q^{1 / 4}=1.6532 \times 10^{-4} \mathrm{~m}
$$

and their mass $m_{G}$ is close to the Planck mass $M_{P}=2.17647 \times 10^{-8} \mathrm{~kg}$ :

$$
m_{G} \sim\left(10^{-9} \Leftrightarrow 10^{-7}\right) \mathrm{kg}
$$

The density of grains $\rho_{G}$ is about:

$$
\rho_{G} \sim \frac{6}{\pi} \frac{M_{P}}{L_{F}^{3}} \approx 9.2 \times 10^{3} \mathrm{~kg} / \mathrm{m}^{3}
$$

According to WUM, Planck mass $M_{P}$ equals to [15]

$$
M_{P}=2 m_{0} \times Q^{1 / 2}
$$

Note that the value of $M_{P}$ is increasing with cosmological time, and is proportional to $\tau^{1 / 2}$. Then,

$$
\frac{\mathrm{d}}{\mathrm{d} \tau} M_{P}=\frac{M_{P}}{2 \tau}
$$

A grain of mass $B_{1} M_{P}$ and radius $B_{2} L_{F}$ is receiving energy from the Medium of the World as the result of dineutrinos Bose-Einstein Condensation (see Section 8 ) at the following rate:

$$
\frac{\mathrm{d}}{\mathrm{d} \tau}\left(B_{1} M_{P} c^{2}\right)=\frac{B_{1} M_{P} c^{2}}{2 \tau}
$$

where $B_{1}$ and $B_{2}$ are parameters.

The received energy will increase the grain's temperature $T_{G}$, until equilibrium is achieved: power received equals to the power irradiated by the surface of a grain in accordance with the Stefan-Boltzmann law

$$
\frac{B_{1} M_{P} c^{2}}{2 \tau}=\sigma_{S B} T_{G}^{4} \times 4 \pi B_{2}^{2} L_{F}^{2}
$$


where $\sigma_{S B}$ is Stefan-Boltzmann constant:

$$
\sigma_{S B}=\frac{2 \pi^{5} k_{B}^{4}}{15 h^{3} c^{2}}
$$

With Nikola Tesla's principle at heart-There is no energy in matter other than that received from the environment-we apply the World equation [23] to a grain:

$$
B_{1} M_{P} c^{2}=4 \pi B_{2}^{2} L_{F}^{2} \sigma_{0}
$$

where $\sigma_{0}$ is a basic unit of surface energy density:

$$
\sigma_{0}=\rho_{0} a
$$

We then calculate the grain's stationary temperature $T_{G}$ to be

$$
T_{G}=\left(\frac{15}{4 \pi^{5}}\right)^{1 / 4} \frac{h c}{k_{B} L_{F}}=28.955 \mathrm{~K}
$$

This result is in an excellent agreement with experimentally measured value of $29 \mathrm{~K}[24]$-[35] and proves the assumptions (7.1), (7.2) and (7.9).

Cosmic FIRB radiation is not a black body radiation. Otherwise, its energy density $\rho_{\text {FIRB }}$ at temperature $T_{G}$ would be too high and equal to the energy density of the Medium of the World:

$$
\rho_{\text {FIRB }}=\frac{8 \pi^{5}}{15} \frac{k_{B}^{4}}{(h c)^{3}} T_{G}^{4}=\frac{2}{3} \rho_{c r}=\rho_{M}
$$

The total flux of the FIRB radiation is the sum of the contributions of all individual grains. Comparing Equations (7.11) and (4.8), we can find the relation between the grains' temperature and the temperature of the MBR:

$$
T_{G}=\left(3 \Omega_{e}\right)^{-1 / 4} \times T_{M B R}
$$

where electron relative energy density $\Omega_{e}$ in terms of the critical energy density equals to

$$
\Omega_{e}=\frac{m_{e}}{m_{p}} \Omega_{p}
$$

\section{Bose-Einstein Condensate}

New cosmological models employing the Bose-Einstein Condensates (BEC) have been actively discussed in literature in recent years [36]-[50]. The transition to BEC occurs below a critical temperature $T_{c}$, which for a uniform three-dimensional gas consisting of non-interacting particles with no apparent internal degrees of freedom is given by

$$
T_{c}=[\zeta(3 / 2)]^{-2 / 3} \frac{h^{2} n_{X}^{2 / 3}}{2 \pi m_{X} k_{B}} \approx \frac{h^{2} n_{X}^{2 / 3}}{11.918 m_{X} k_{B}}
$$

where $n_{X}$ is the particle concentration, $m_{X}$ is the mass per boson, $\zeta$ is the Riemann zeta function:

$$
\zeta(3 / 2) \approx 2.6124
$$


According to our Model, we can take the value of the critical temperature $T_{c}$ to equal the stationary temperature $T_{G}$ of Large Grains (see Equation (7.11)). Let's assume that the energy density of boson particles $\rho_{X}$ equals to the MBR energy density (see (4.7)):

$$
\rho_{X}=n_{X} m_{X}=2 \frac{m_{e}}{m_{p}} \rho_{p}=4 \pi^{2} \alpha \frac{m_{e}}{m_{p}} \frac{h c}{L_{F}^{4}}=1.5690 \times 10^{-4} \times \frac{h c}{L_{F}^{4}}
$$

Taking into account Equations (7.11), (8.1) and (8.3), we can calculate the value of $n_{X}$ :

$$
\begin{aligned}
n_{X} & =\left[47.672 \pi^{2} \alpha \frac{m_{e}}{m_{p}}\left(\frac{15}{4 \pi^{5}}\right)^{1 / 4}\right]^{3 / 5} \times L_{F}^{-3} \\
& =0.011922 \times L_{F}^{-3}=2.6386 \times 10^{9} \mathrm{~m}^{-3}
\end{aligned}
$$

and the value of the mass $m_{X}$ :

$$
m_{X}=\frac{\rho_{X}}{n_{X} c^{2}}=0.013161 \times m_{0} \times Q^{-1 / 4}=0.987 \times 10^{-4} \mathrm{eV} / \mathrm{c}^{2}
$$

$m_{X}$ is about 10 orders of magnitude larger than the rest mass of photon's (see (4.5)) and is in the range of neutrinos masses (see Section 6).

The calculated values of mass and concentration of dineutrinos satisfy the conditions for their Bose-Einstein condensation. Consequently, BEC drops whose masses are about Planck mass can be created. The stability of such drops is provided by the detailed equilibrium between the energy absorption from the Medium of the World (provided by dineutrinos as a result of their Bose-Einstein condensation) and re-emission of this energy in FIRB at the stationary temperature $T_{G} \approx 29 \mathrm{~K}$ (see Section 7 ).

In WUM the FIRB energy density $\rho_{\text {FIRB }}$ equals to [15]

$$
\rho_{\text {FIRB }}=\frac{1}{5 \pi} \frac{m_{e}}{m_{p}} \rho_{p}=\frac{2 \pi \alpha}{15} \frac{m_{e}}{m_{p}}
$$

which is $10 \pi$ times smaller than the energy density of MBR and dineutrinos:

$$
\rho_{\text {FIRB }}=\frac{1}{10 \pi} \rho_{\text {MBR }} \approx 0.032 \rho_{\text {MBR }}
$$

The ratio between FIRB and MBR corresponds to the value of $3.4 \%$ calculated by E. L. Wright [51].

\section{Multicomponent Dark Matter}

Dark Matter (DM) is among the most important open problems in both cosmology and particle physics. Dark Matter problem can be, in principle, achieved through extended theories of gravity, as it is discussed, for example, in [52].

There are three prominent hypotheses on nonbaryonic DM, namely Hot Dark Matter (HDM), Warm Dark Matter (WDM), and Cold Dark Matter (CDM). A neutralino with mass $m_{N}$ in $100 \Leftrightarrow 10000 \mathrm{GeV} / c^{2}$ range is the leading CDM candidate. Light DMP is heavier than WDM and HDM but lighter than neutralinos are DM candidates too. Subsequently, we will refer to the light DMP as 
WIMPs. Their mass $m_{\text {WIMP }}$ falls into $1 \Leftrightarrow 10 \mathrm{GeV} / \mathrm{c}^{2}$ range. It is known that a sterile neutrino with mass $m_{v_{s}}$ in $1 \Leftrightarrow 10 \mathrm{keV} / \mathrm{c}^{2}$ range is a good WDM candidate. In our opinion, a tauonic neutrino is a good HDM candidate.

In addition to fermions discussed above, we offer another type of DMP-bosons, consisting of two fermions each. There exist two types of DM bosons which we called DIRACs and ELOPs [23]. DIRACs are magnetic dipoles with mass $m_{0}$, consisting of two Dirac monopoles with mass about $\frac{m_{0}}{2}$ and charge $\mu=\frac{e}{2 \alpha}$. Dissociated DIRACs can only exist at nuclear densities or at high temperatures. In our opinion, Dirac monopoles are the smallest building blocks of constituent quarks and hadrons (mesons and baryons).

The second boson is the ELOP (named by analogy to an ELectron-nortisOP dipole). ELOP weighs $\frac{2}{3} m_{e}$ and consists of two preons with mass $m_{p r}=\frac{1}{3} m_{e}$ and charge $e_{p r}=\frac{1}{3} e$ which we took to match the Quark Model. ELOPs break into two preons at nuclear densities or at high temperatures. In particle physics, preons are postulated to be "point-like" particles, conceived to be subcomponents of quarks and leptons [53].

WUM postulates that masses of DMP are proportional to $m_{0}$ multiplied by different exponents of $\alpha$ and can be expressed with the following formulae:

CDM particles (neutralinos and WIMPs):

$$
\begin{gathered}
m_{N}=\alpha^{-2} m_{0}=1.3149950 \mathrm{TeV} / \mathrm{c}^{2} \\
m_{\text {WIMP }}=\alpha^{-1} m_{0}=9.5959823 \mathrm{GeV} / \mathrm{c}^{2}
\end{gathered}
$$

DIRACs:

$$
m_{\text {DIRAC }}=2 \alpha^{0} \frac{m_{0}}{2}=70.025267 \mathrm{MeV} / \mathrm{c}^{2}
$$

ELOPs:

$$
m_{E L O P}=2 \alpha^{1} \frac{m_{0}}{3}=340.66606 \mathrm{keV} / \mathrm{c}^{2}
$$

WDM particles (sterile neutrinos):

$$
m_{v_{s}}=\alpha^{2} m_{0}=3.7289402 \mathrm{keV} / \mathrm{c}^{2}
$$

These values fall into the ranges estimated in literature. The role of those particles in macroobject cores built up from fermionic dark matter will be discussed in Section 10.

Our Model holds that the energy densities of all types of DMP are proportional to the proton energy density $\rho_{p}$ in the World's Medium (see (4.6)). In all, there are 5 different types of DMP. Then the total energy density of DMP is

$$
\rho_{D M}=5 \rho_{p}=0.24007327 \rho_{c r}
$$

which is close to the measured DM energy density: $\rho_{D M} \cong 0.268 \rho_{\text {cr }}$ [54]. Note that one of outstanding puzzles in particle physics and cosmology relates to 
so-called cosmic coincidence: the ratio of dark matter density in the World to baryonic matter density in the Medium of the World $\cong 5$ [55] [56].

Neutralinos, WIMPs, and sterile neutrinos are Majorana fermions, which partake in the annihilation interaction with strength equals to $\alpha^{-2}, \alpha^{-1}$, and $\alpha^{2}$ respectively (see Section 10). The signatures of DMP annihilation with expected masses of $1.3 \mathrm{TeV}, 9.6 \mathrm{GeV}, 70 \mathrm{MeV}, 340 \mathrm{keV}$, and $3.7 \mathrm{keV}$ are found in spectra of the diffuse gamma-ray background and the emission of various macroobjects in the World [23].

The assumptions made in (8.3) and (8.6) are further supported by the excellent numerical agreement of calculated and measured value of fine-structure constant $\alpha$ discussed in Section 11.

\section{Macroobject Cores Built up from Fermionic Dark Matter}

In this section, we discuss the possibility of all macroobject cores consisting of DMP introduced in Section 9. The first phase of stellar evolution in the history of the World may be dark stars, powered by Dark Matter heating rather than fusion. Neutralinos and WIMPs, which are their own antiparticles, can annihilate and provide an important heat source for the stars and planets in the World.

In our view, all macroobjects of the World (including galaxy clusters, galaxies, star clusters, extrasolar systems, and planets) possess the following properties:

- Macroobject cores are made up of DMP;

- Macroobjects consist of all particles under consideration, in the same proportion as they exist in the World's Medium;

- Macroobjects contain other particles, including DM and baryonic matter, in shells surrounding the cores.

Taking into account the main principle of the World-Universe Model (all physical parameters can be expressed in terms of $\alpha, Q$, small integer numbers, and $\pi$ ) we modify the published theory of Fermionic Compact Stars (FCS) developed by G. Narain, et al. [57] as follows. We take a scaling solution for a free Fermi gas consisting of fermions with mass $m_{f}$ in accordance with following equations:

$$
\begin{aligned}
& \text { Maximum mass: } M_{\max }=A_{1} M_{F} \text {; } \\
& \text { Minimum radius: } R_{\min }=A_{2} R_{F} ; \\
& \text { Maximum density: } \rho_{\max }=A_{3} \rho_{0}
\end{aligned}
$$

where

$$
M_{F}=\frac{M_{P}^{3}}{m_{f}^{2}} ; R_{F}=\frac{M_{P}}{m_{f}} \frac{L_{C f}}{2 \pi} ; \rho_{0}=\frac{h c}{a^{4}}
$$

and $M_{P}$ is Planck mass, $L_{C f}$ is a Compton length of the fermion. $A_{1}, A_{2}$, and $A_{3}$ are parameters. Let us choose $\pi$ as the value of $A_{2}$ (instead of $A_{2}=3.367$ taken by G. Narain, et al. [57]). Then diameter of FCS is proportional to the fermion Compton length $L_{C f}$. We use $\pi / 6$ as the value of $A_{1}$ (instead of $A_{1}=0.384$ taken by G. Narain, et al. [57]). Then $A_{3}$ will equal to

$$
A_{3}=\left(\frac{m_{f}}{m_{0}}\right)^{4}
$$


Table 1. Parameter values for FCS made up of various fermions.

\begin{tabular}{ccccc}
\hline Fermion & $\begin{array}{c}\text { Fermion } \\
\text { relative mass } \\
m_{f} / m_{0}\end{array}$ & $\begin{array}{c}\text { Macroobject } \\
\text { relative mass } \\
M_{\max } / M_{0}\end{array}$ & $\begin{array}{c}\text { Macroobject } \\
\text { relative radius } \\
R_{\min } / L_{g}\end{array}$ & $\begin{array}{c}\text { Macroobject } \\
\text { relative density } \\
\rho_{\max } / \rho_{0}\end{array}$ \\
\hline $\begin{array}{c}\text { Sterile neutrino } \\
\text { Preon }\end{array}$ & $\alpha^{2}$ & $\alpha^{-4}$ & $\alpha^{-4}$ & $\alpha^{8}$ \\
$\begin{array}{c}3^{-1} \alpha^{1} \\
\text { Electron-proton }\end{array}$ & $3^{2}, \beta$ & $3^{2}$ & $3^{2} \alpha^{-2}$ & $3^{-4} \alpha^{4}$ \\
(white dwarf) & $2^{-1}$ & $(\alpha \beta)^{-1}$ & $\alpha^{3} \beta$ \\
Monopole & $\alpha^{-1}$ & $2^{2}$ & $2^{2}$ & $2^{-4}$ \\
WiMP & $\alpha^{-2}$ & $\alpha^{2}$ & $\alpha^{2}$ & $\alpha^{-4}$ \\
Interacting WIMPs & $\alpha^{-1}$ & $\beta^{-2}$ & $\alpha^{4}$ & $\alpha^{-8}$ \\
Interacting & $\alpha^{-2}$ & $\beta^{-2}$ & $\beta^{-2}$ & $\beta^{4}$ \\
neutralinos & & $\beta^{-2}$ & $\beta^{-2}$ & $\beta^{4}$ \\
Neutron (star) & $\approx \beta$ & & & $\beta^{4}$ \\
\hline
\end{tabular}

Table 1 summarizes the parameter values for FCS made up of various fermions:

where $M_{0}=\frac{4 \pi m_{0}}{3} \times Q^{3 / 2}$

$$
\begin{gathered}
L_{g}=a \times Q^{1 / 2} \\
\beta=\frac{m_{p}}{m_{0}}
\end{gathered}
$$

A maximum density of neutron stars equals to the nuclear density:

$$
\rho_{\max }=\beta^{4} \rho_{0}
$$

which is the maximum possible density of any macroobject in the World.

A Compact Star made up of heavier particles, WIMPs and neutralinos, could in principle have a much higher density. In order for such a star to remain stable and not exceed the nuclear density, WIMPs and neutralinos must partake in an annihilation interaction whose strength equals to $\alpha^{-1}$ and $\alpha^{-2}$ respectively.

Scaling solution for interacting WIMPs can also be described with equations (10.1), (10.2), (10.3) and the following values of $A_{1}, A_{2}$ and $A_{3}$ :

$$
\begin{gathered}
A_{1 \text { max }}=\frac{\pi}{6}(\alpha \beta)^{-2} \\
A_{2 \text { min }}=\pi(\alpha \beta)^{-2} \\
A_{3 \text { max }}=\beta^{4}
\end{gathered}
$$

The maximum mass and minimum radius increase about two orders of magnitude each and the maximum density equals to the nuclear density. Note that parameters of a FCS made up of strongly interacting WIMPs are identical to those of neutron stars.

In accordance with the paper by G. Narain, et al. [57], the most attractive 
feature of the strongly interacting Fermi gas of WIMPs is practically constant value of FCS minimum radius in the large range of masses $M_{\text {WIMP }}$ from

$$
M_{\text {WIMP max }}=\frac{\pi}{6}(\alpha \beta)^{-2} M_{F}=\frac{1}{\beta^{2}} M_{0}
$$

down to

$$
M_{\text {WIMP } \min }=\alpha^{4} M_{\text {WIMP } \max }
$$

$M_{\text {WIMP min }}$ is more than eight orders of magnitude smaller than $M_{\text {WIMP max }}$. It makes strongly interacting WIMPs good candidates for stellar and planetary cores of extrasolar systems with Red stars [23].

When the mass of a FCS made up of WIMPs is much smaller than the maximum mass, the scaling solution yields the following equation for parameters $A_{1}$ and $A_{2}$ :

$$
A_{1} A_{2}^{3}=\pi^{4}
$$

Compare $\pi^{4} \cong 97.4$ with the value of 91 used by G. Narain, et al. [57].

Minimum mass and maximum radius take on the following values:

$$
\begin{aligned}
& A_{1 \min }=\frac{\pi}{6} \sqrt{6}(\alpha \beta)^{2} \\
& A_{2 \max }=\pi \sqrt[6]{6}(\alpha \beta)^{-2 / 3}
\end{aligned}
$$

It follows that the range of FCS masses $\left(A_{1 \min } \Leftrightarrow A_{1 \max }\right)$ spans about three orders of magnitude, and the range of FCS core radii $\left(A_{2 \min } \Leftrightarrow A_{2 \max }\right)$-one order of magnitude. It makes WIMPs good candidates for brown dwarf cores too [23].

Scaling solution for interacting neutralinos can be described with the same equations (10.1), (10.2), (10.3) and the following values of $A_{1}^{*}, A_{2}^{*}$ and $A_{3}^{*}$ :

$$
\begin{gathered}
A_{1 \text { max }}^{*}=\frac{\pi}{6}\left(\alpha^{2} \beta\right)^{-2} \\
A_{2 \min }^{*}=\pi\left(\alpha^{2} \beta\right)^{-2} \\
A_{3 \text { max }}^{*}=\beta^{4}
\end{gathered}
$$

In this case, the maximum mass and minimum radius increase about four orders of magnitude each and the maximum density equals to the nuclear density. Note that parameters of a FCS made up of strongly interacting neutralinos are identical to those of neutron stars.

Practically constant value of FCS minimum radius takes place in the huge range of masses $M_{N}$ from

$$
M_{N \max }=\frac{\pi}{6}(\alpha \beta)^{-2} \alpha^{2} M_{F}=\frac{1}{\beta^{2}} M_{0}
$$

down to

$$
M_{N \text { min }}=\alpha^{8} M_{N \text { max }}
$$

$M_{N \text { min }}$ is more than seventeen orders of magnitude smaller than $M_{N \max }$. It makes strongly interacting neutralinos good candidates for stellar and planetary cores of extrasolar systems with Main-sequence stars [23]. 
When the mass of a FCS made up of neutralinos is much smaller than the maximum mass, the scaling solution yields the following equation for parameters $A_{1}^{*}$ and $A_{2}^{*}$ :

$$
A_{1}^{*} A_{2}^{* 3}=\pi^{4}
$$

Minimum mass and maximum radius take on the following values:

$$
\begin{aligned}
A_{1 \text { min }}^{*} & =\frac{\pi}{6} \sqrt{6}\left(\alpha^{2} \beta\right)^{2} \\
A_{2 \max }^{*} & =\pi \sqrt[6]{6}\left(\alpha^{2} \beta\right)^{-2 / 3}
\end{aligned}
$$

It means that the range of FCS masses $\left(A_{1 \min }^{*} \Leftrightarrow A_{1 \max }^{*}\right)$ is about twelve orders of magnitude, and the range of FCS core radiuses $\left(A_{2 \min }^{*} \Leftrightarrow A_{2 \max }^{*}\right)$ is about four orders of magnitude.

Fermionic Compact Stars have the following properties:

- The maximum potential of interaction $U_{\max }$ between any particle or macroobject and FCS made up of any fermions

$$
U_{\max }=\frac{G M_{\max }}{R_{\min }}=\frac{c^{2}}{6}
$$

does not depend on the nature of fermions;

- The minimum radius of FCS made of any fermion

$$
R_{\min }=3 R_{S H}
$$

equals to three Schwarzschild radii and does not depend on the nature of the fermion;

- FCS density does not depend on $M_{\max }$ and $R_{\min }$ and does not change in time while $M_{\max } \propto \tau^{3 / 2}$ and $R_{\min } \propto \tau^{1 / 2}$.

\section{Energy Density of Dineutrinos, FIRB and the World}

Our Model holds that the energy densities of all types of Dark Matter particles (DMP) are proportional to the proton energy density in the World's Medium. In all, there are 5 different types of DMP (see Section 9). Then the total energy density of Dark Matter (DM) $\Omega_{D M}$ is

$$
\Omega_{D M}=5 \Omega_{p}
$$

The total electron energy density $\Omega_{\text {etot }}$ is:

$$
\Omega_{\text {etot }}=1.5 \frac{m_{e}}{m_{p}} \Omega_{p}
$$

The MBR energy density $\Omega_{M B R}$ equals to [1]:

$$
\Omega_{\text {MBR }}=2 \frac{m_{e}}{m_{p}} \Omega_{p}
$$

We took energy density of dineutrinos $\Omega_{v \bar{v}}$ and FIRB $\Omega_{F I R B}$ (see Section 8):

$$
\Omega_{v \bar{v}}=\Omega_{M B R}=2 \frac{m_{e}}{m_{p}} \Omega_{p}
$$




$$
\Omega_{F I R B}=\frac{1}{5 \pi} \frac{m_{e}}{m_{p}} \Omega_{p}=\frac{1}{10 \pi} \Omega_{M B R} \approx 0.032 \Omega_{M B R}
$$

Then the energy density of the World $\Omega_{W}$

$$
\Omega_{W}=\left[\frac{13}{2}+\left(\frac{11}{2}+\frac{1}{5 \pi}\right) \frac{m_{e}}{m_{p}}+\frac{45}{\pi}\right] \Omega_{p}=1
$$

Equation (11.6) contains such exact terms as the result of the Models' predictions and demonstrates consistency of WUM. From (11.6) we can calculate the value of $\alpha$, using electron-to-proton mass ratio $\frac{m_{e}}{m_{p}}$

$$
\frac{1}{\alpha}=\frac{\pi}{15}\left[450+65 \pi+(55 \pi+2) \frac{m_{e}}{m_{p}}\right]=137.03600
$$

which is in an excellent agreement with the commonly adopted value of 137.035999074 (44). It follows that there exists a direct correlation between constants $\alpha$ and $\frac{m_{e}}{m_{p}}$ expressed by Equation (11.6). As shown above, $\frac{m_{e}}{m_{p}}$ is not an independent constant, but is instead derived from $\alpha$.

\section{Grand Unified Theory}

At the very Beginning $(Q=1)$ all extrapolated fundamental interactions of the World-strong, electromagnetic, weak, Super Weak and Extremely Weak (proposed in WUM), and gravitational-had the same cross-section of $\left(\frac{\pi a}{2}\right)^{2}$, and could be characterized by the Unified coupling constant: $\alpha_{U}=1$. The extrapolated energy density of the World was four orders of magnitude smaller than the nuclear energy density [1]. The average energy density of the World has since been decreasing in time $\rho_{W} \propto Q^{-1} \propto \tau^{-1}$.

The gravitational coupling parameter $\alpha_{G}$ is similarly decreasing:

$$
\alpha_{G}=Q^{-1} \propto \tau^{-1}
$$

The weak coupling parameter $\alpha_{W}$ is decreasing as follows:

$$
\alpha_{W}=Q^{-1 / 4} \propto \tau^{-1 / 4}
$$

The strong $\alpha_{S}$ and electromagnetic $\alpha_{E M}$ coupling parameters remain constant in time:

$$
\alpha_{S}=\alpha_{E M}=1
$$

The difference in the strong and the electromagnetic interactions is not in the coupling parameters but in the strength of these interactions depending on the particles involved: electrons with charge $e$ and monopoles with charge $\mu=\frac{e}{2 \alpha}$ in electromagnetic and strong interactions respectively.

The super weak coupling parameter $\alpha_{S W}$ and the extremely weak coupling parameter $\alpha_{E W}$ proposed in WUM are decreasing as follows: 


$$
\begin{gathered}
\alpha_{S W}=Q^{-1 / 2} \propto \tau^{-1 / 2} \\
\alpha_{E W}=Q^{-3 / 4} \propto \tau^{-3 / 4}
\end{gathered}
$$

According to WUM, the coupling strength of super-weak interaction is $\sim 10^{-10}$ times weaker than that of weak interaction. The possibility of such ratio of interactions was discussed in the developed theoretical models explaining $\mathrm{CP}$ and Strangeness violation [58] [59] [60] [61]. Super-weak and Extremely-weak interactions provide an important clue to Physics beyond the Standard Model.

\section{Conclusions}

WUM holds that there exist relations between all $Q$-dependent parameters: Newtonian parameter of gravitation and Hubble's parameter; critical energy density and Fermi coupling parameter; temperatures of the microwave background radiation and far-infrared background radiation peak. The calculated values of these parameters are in good agreement with the latest results of their measurements.

Today, Fermi coupling parameter $G_{F}$ is known with the highest precision [1]:

$$
\frac{G_{F}}{(\hbar c)^{3}}=\sqrt{30}\left(2 \alpha \frac{m_{e}}{m_{p}}\right)^{1 / 4} \times \frac{m_{p}}{m_{e}} \frac{1}{E_{0}^{2}} \times Q^{-1 / 4}
$$

Based on its average value, we can calculate and significantly increase the precision of all $Q$-dependent parameters. We propose to introduce $Q$ as a new fundamental parameter tracked by CODATA, and use its value in calculation of all $Q$-dependent parameters.

\section{Acknowledgements}

I am grateful to anonymous referees for valuable comments and important remarks that helped me to improve the understanding of the Model. Special thanks to my son Ilya Netchitailo who helped shape the manuscript to its present form.

\section{References}

[1] Netchitailo, V.S. (2016) Overview of Hypersphere World-Universe Model. Journal of High Energy Physics, Gravitation and Cosmology, 2, 593. https://doi.org/10.4236/jhepgc.2016.24052

[2] Netchitailo, V.S. (2015) 5D World-Universe Model. Space-Time-Energy. Journal of High Energy Physics, Gravitation and Cosmology, 1, 25. https://doi.org/10.4236/jhepgc.2015.11003

[3] Netchitailo, V.S. (2016) 5D World-Universe Model. Gravitation. Journal of High Energy Physics, Gravitation and Cosmology, 2, 328. https://doi.org/10.4236/jhepgc.2016.23031

[4] Netchitailo, V.S. (2017) Burst Astrophysics. Journal of High Energy Physics, Gravitation and Cosmology, 3, 157-166. https://doi.org/10.4236/jhepgc.2017.32016

[5] Bennett, C.L., et al. (2013) Nine-Year Wilkinson Microwave Anisotropy Probe 
(WMAP) Observations: Final Maps and Results.

[6] Mirizzi, A., Raffelt, G.G. and Serpico, P.D. (2006) Photon-Axion Conversion in Intergalactic Magnetic Fields and Cosmological Consequences.

[7] Spergel, D.N., et al. (2003) First Year Wilkinson Microwave Anisotropy Probe (WMAP) Observations: Determination of Cosmological Parameters.

[8] Bonetti, L., et al. (2017) FRB 121102 Casts New Light on the Photon Mass.

[9] Keane, E.F., et al. (2016) A Fast Radio Burst Host Galaxy.

[10] Fixsen, D.J. (2009) The Temperature of the Cosmic Microwave Background. http://arxiv.org/abs/0911.1955 https://doi.org/10.1088/0004-637x/707/2/916

[11] Masui, K., et al. (2015) Dense Magnetized Plasma Associated with a Fast Radio Burst. Nature, 528, 523. https://doi.org/10.1038/nature15769

[12] Lorimer, D.R., et al. (2007) A Bright Millisecond Radio Burst of Extragalactic Origin. Science, 318, 777. https://doi.org/10.1126/science.1147532

[13] Kajita, T. (1998) Atmospheric Neutrino Results from Super-Kamiokande and Kamiokande-Evidence for $\nu \mu$ Oscillations.

[14] McDonald, A.B. (2003) Neutrino Properties from Measurements Using Astrophysical and Terrestrial Sources.

[15] Netchitailo, V.S. (2016) 5D World-Universe Model. Neutrinos. The World. Journal of High Energy Physics, Gravitation and Cosmology, 2, 1. https://doi.org/10.4236/jhepgc.2016.21001

[16] Sanchez, M. (2003) Oscillation Analysis of Atmospheric Neutrinos in Soudan 2. PhD Thesis, Tufts University, Medford/Somerville. http://nu.physics.iastate.edu/Site/Bio_files/thesis.pdf https://doi.org/10.2172/815661

[17] Kaus, P. and Meshkov, S. (2003) Neutrino Mass Matrix and Hierarchy. AIP Conference Proceedings, 672, 117. https://doi.org/10.1063/1.1594399

[18] Battye, R.A. and Moss, A. (2014) Evidence for Massive Neutrinos from CMB and Lensing Observations.

[19] Landau, L.D. and Lifshitz, E.M. (1980) Statistical Physics. 3rd Edition, Part 1, Vol. 5, Butterworth-Heinemann, Oxford.

[20] Maurette, M., Cragin, J. and Taylor, S. (1992) Cosmic Dust in 50 KG Blocks of Blue Ice from Cap-Prudhomme and Queen Alexandra Range, Antarctica. Meteoritics, 27, 257.

[21] Saxton, J.M., Knotts, S.F., Turner, G. and Maurette, M. (1992) 40Ar/39Ar Studies of Antarctic Micrometeorites. Meteoritics, 27, 285.

[22] Jackson, A.A. and Zook, H.A. (1991) Dust Particles from Comets and Asteroids: Parent-Daughter Relationships. Abstracts of the Lunar and Planetary Science Conference, 22, 629-630.

[23] Netchitailo, V.S. (2015) 5D World-Universe Model. Multicomponent Dark Matter. Journal of High Energy Physics, Gravitation and Cosmology, 1, 55-71. https://doi.org/10.4236/jhepgc.2015.12006

[24] Lagache, G., Abergel, A., Boulanger, F., Désert, F.X. and Puget, J.-L. (1999) First Detection of the Warm Ionized Medium Dust Emission. Implication for the Cosmic Far-Infrared Background. Astronomy and Astrophysics, 344, 322-332.

[25] Finkbeiner, D.P., Davis, M. and Schlegel, D.J. (2000) Detection of a Far IR Excess with DIRBE at 60 and 100 Microns. The Astrophysical Journal, 544, 81-97. https://doi.org/10.1086/317177 
[26] Siegel, P.H. (2002) Terahertz Technology. IEEE Transactions on Microwave Theory and Techniques, 50, 910-928. https://doi.org/10.1109/22.989974

[27] Phillips, T.G. and Keene, J. (1992) Submillimeter Astronomy [Heterodyne Spectroscopy]. Proceedings of the IEEE, 80, 1662-1678. https://doi.org/10.1109/5.175248

[28] Dupac, X., et al. (2003) The Complete Submillimeter Spectrum of NGC 891.

[29] Aguirre, J.E., Bezaire, J.J., Cheng, E.S., Cottingham, D.A., Cordone, S.S., Crawford, T.M., et al. (2003) The Spectrum of Integrated Millimeter Flux of the Magellanic Clouds and 30-Doradus from TopHat and DIRBE Data. The Astrophysical Journal, 596, 273-286. https://doi.org/10.1086/377601

[30] Pope, A., Scott, D., Dickinson, M., Chary, R.-R., Morrison, G., Borys, C. and Sajina, A. (2006) Using Spitzer to Probe the Nature of Submillimetre Galaxies in GOODSN.

[31] Marshall, J.A., Herter, T.L., Armus, L., Charmandaris, V., Spoon, H.W.W., BernardSalas, J. and Houck, J.R. (2007) Decomposing Dusty Galaxies. I. Multi-Component Spectral Energy Distribution Fitting. The Astrophysical Journal, 670, 129-155 https://doi.org/10.1086/521588

[32] Devlin, M.J., Ade, P.A.R., Aretxaga, I., Bock, J.J., Chapin, E.L., Griffin, M., et al. (2009) Over Half of the Far-Infrared Background Light Comes from Galaxies at $\mathrm{z} \geq$ 1.2. Nature, 458, 737-739. https://doi.org/10.1038/nature07918

[33] Chapin, E.L., Chapman, S.C., Coppin, K.E., Devlin, M.J., Dunlop, J.S., Greve, T.R., et al. (2011) A Joint Analysis of BLAST 250-500 um and LABOCA 870 um Observations in the Extended Chandra Deep Field-South. Monthly Notices of the Royal Astronomical Society, 411, 505-549. https://doi.org/10.1111/j.1365-2966.2010.17697.x

[34] Mackenzie, T., Braglia, F.G., Gibb, A.G., Scott, D., Jenness, T., Serjeant, S., et al. (2011) A Pilot Study for the SCUBA-2 "All-Sky" Survey. Monthly Notices of the Royal Astronomical Society, 415, 1950-1960. https://doi.org/10.1111/j.1365-2966.2011.18840.x

[35] Serra, P., Lagache, G., Doré, O., Pullen, A. and White, M. (2014) Cross-Correlation of Cosmic Infrared Background Anisotropies with Large Scale Structures. Astronomy \& Astrophysics, 570, A98. https://doi.org/10.1051/0004-6361/201423958

[36] Sin, S.-J. (1992) Late Time Cosmological Phase Transition and Galactic Halo as Bose-Liquid.

[37] Robles, V.H. and Matos, M. (2012) Flat Central Density Profile and Constant DM Surface Density in Galaxies from Scalar Field Dark Matter. Monthly Notices of the Royal Astronomical Society, 422, 282-289. https://doi.org/10.1111/j.1365-2966.2012.20603.x

[38] Magana, J. and Matos, T. (2012) A Brief Review of the Scalar Field Dark Matter Model. Journal of Physics: Conference Series, 378, Article ID: 012012. https://doi.org/10.1088/1742-6596/378/1/012012

[39] Suarez, A., Robles, V.H. and Matos, T. (2013) A Review on the Scalar Field/BoseEinstein Condensate Dark Matter Model. In: González, C.M., Aguilar, J.E.M. and Barrera, L.M.R., Eds., Accelerated Cosmic Expansion, Springer, Berlin, 107-142.

[40] Diez-Tejedor, A., Gonzalez-Morales, A.X. and Profumo, S. (2014) Dwarf Spheroidal Galaxies and Bose-Einstein Condensate Dark Matter. Physical Review D, 90, Article ID: 043517. https://doi.org/10.1103/PhysRevD.90.043517

[41] Sikivie, P. and Yang, Q. (2009) Bose-Einstein Condensation of Dark Matter Axions. Physical Review Letters, 103, Article ID: 111301. https://doi.org/10.1103/physrevlett.103.111301

[42] Erken, O., Sikivie, P., Tam, H. and Yang, Q. (2011) Axion BEC Dark Matter. 
[43] Banik, N. and Sikivie, P. (2013) Axions and the Galactic Angular Momentum Distribution. Physical Review D, 88, Article ID: 123517. https://doi.org/10.1103/PhysRevD.88.123517

[44] Davidson, S. and Elmer, M. (2013) Bose Einstein Condensation of the Classical Axion Field in Cosmology? Journal of Cosmology and Astroparticle Physics, 2013, Article No. 034. https://doi.org/10.1088/1475-7516/2013/12/034

[45] Li, M.-H. and Li, Z.-B. (2014) Constraints on Bose-Einstein-Condensed Axion Dark Matter from the HI nearby Galaxy Survey Data. Physical Review D, 89, Article ID: 103512. https://doi.org/10.1103/physrevd.89.103512

[46] Morikawa, M. (2004) Structure Formation through Cosmic Bose Einstein Condensation-Unified View of Dark Matter and Energy. 22nd Texas Symposium on Relativistic Astrophysics, Stanford, 13-17 December 2004, 1122.

[47] Garay, L.J., Anglin, J.R., Cirac, J.I. and Zoller, P. (2000) Sonic Analog of Gravitational Black Holes in Bose-Einstein Condensates. Physical Review Letters, 85, 4643 4647. https://doi.org/10.1103/PhysRevLett.85.4643

[48] Ueda, M. and Huang, K. (1998) Fate of a Bose-Einstein Condensate with Attractive Interaction.

[49] Hujeirat, A.A. (2011) On the Viability of Gravitational Bose-Einstein Condensates as Alternatives to Supermassive Black Holes. Monthly Notices of the Royal Astronomical Society, 423, 2893-2900. https://doi.org/10.1111/j.1365-2966.2012.21102.x

[50] Kuhnel, F. and Sundborg, B. (2014) Decay of Graviton Condensates and Their Generalizations in Arbitrary Dimensions. Physical Review D, 90, Article ID: 064025. https://doi.org/10.1103/PhysRevD.90.064025

[51] Wright, E.L. (2001) Cosmic InfraRed Background Radiation. http://www.astro.ucla.edu/ wright/CIBR/

[52] Corda, C. (2009) Interferometric Detection of Gravitational Waves: The Definitive Test for General Relativity. International Journal of Modern Physics D, 18, 2275. https://doi.org/10.1142/s0218271809015904

[53] D’Souza, I.A. and Kalman, C.S. (1992) Preons: Models of Leptons, Quarks and Gauge Bosons as Composite Objects. World Scientific, Singapore. https://doi.org/10.1142/1700

[54] NASA's Planck Project Office (2013) Planck Mission Brings Universe into Sharp Focus. https://www.nasa.gov/mission_pages/planck/news/planck20130321.html\#.VZ4k5_l $\underline{\text { Viko }}$

[55] Feng, W.Z., Mazumdar, A. and Nath, P. (2013) Baryogenesis from Dark Matter. Physical Review D, 88, Article ID: 036014.

http://arxiv.org/abs/1302.0012 https://doi.org/10.1103/physrevd.88.036014

[56] Feng, W.Z., Nath, P. and Peim, G. (2012) Cosmic Coincidence and Asymmetric Dark Matter in a Stueckelberg Extension. Physical Review D, 85, Article ID: 115016. http://arxiv.org/abs/1204.5752 https://doi.org/10.1103/physrevd.85.115016

[57] Narain, G., Schaffner-Bielich, J. and Mishustin, I.N. (2006) Compact Stars Made of Fermionic Dark Matter. Physical Review D, 74, Article ID: 063003.

http://arxiv.org/abs/astro-ph/0605724 https://doi.org/10.1103/physrevd.74.063003

[58] Wolfenstein, L. (1994) Superweak Interactions. Comments on Nuclear and Particle Physics, 21, 275. 
[59] Yamaguchi, Y. (1959) Possibility of Super-Weak Interactions and the Stability of Matter. Progress of Theoretical Physics, 22, 373. https://doi.org/10.1143/PTP.22.373

[60] Kelley, K.F. (1999) Measurement of the CP Violation Parameter $\sin 2 \beta$. PhD Thesis, MIT.

[61] Bian, B.A., et al. (2006) Determination of the NN Cross Section, Symmetry Energy, and Studying of Weak Interaction in CSR.

http://ribll.impcas.ac.cn/conf/ccast05/doc/RIB05-zhangfengshou.pdf

Submit or recommend next manuscript to SCIRP and we will provide best service for you:

Accepting pre-submission inquiries through Email, Facebook, LinkedIn, Twitter, etc. A wide selection of journals (inclusive of 9 subjects, more than 200 journals)

Providing 24-hour high-quality service

User-friendly online submission system

Fair and swift peer-review system

Efficient typesetting and proofreading procedure

Display of the result of downloads and visits, as well as the number of cited articles

Maximum dissemination of your research work

Submit your manuscript at: http://papersubmission.scirp.org/

Or contact jhepgc@scirp.org 